\title{
THE BEST CONSTANT OF THREE KINDS OF THE DISCRETE SOBOLEV INEQUALITIES ON THE COMPLETE GRAPH
}

\author{
Hiroyuki Yamagishi, Kohtaro Watanabe and Yoshinori Kametaka
}

\begin{abstract}
We introduce a discrete Laplacian $\boldsymbol{A}$ on the complete graph with $N$ vertices, that is, $K_{N}$. We obtain the best constants of three kinds of discrete Sobolev inequalities on $K_{N}$. The background of the first inequality is the discrete heat operator $\left(d / d t+\boldsymbol{A}+a_{0} \boldsymbol{I}\right) \cdots\left(d / d t+\boldsymbol{A}+a_{M-1} \boldsymbol{I}\right)$ with positive distinct characteristic roots $a_{0}, \ldots, a_{M-1}$. The second one is the difference operator $\left(\boldsymbol{A}+a_{0} \boldsymbol{I}\right) \cdots\left(\boldsymbol{A}+a_{M-1} \boldsymbol{I}\right)$ and the third one is the discrete polyharmonic operator $\boldsymbol{A}^{M}$. Here $\boldsymbol{A}$ is an $N \times N$ real symmetric positive-semidefinite matrix whose eigenvector corresponding to zero eigenvalue is $\mathbf{1}={ }^{t}(1,1, \ldots, 1)$. A discrete heat kernel, a Green's matrix and a pseudo Green's matrix are obtained by means of $\boldsymbol{A}$.
\end{abstract}

\section{Introduction}

For any fixed $M=1,2,3, \ldots$, we put $a=\left(a_{0}, \ldots, a_{M-1}\right)$ and assume $0<a_{0}<a_{1}<\cdots<a_{M-1}$. We introduce the characteristic polynomial

$$
P(z)=\prod_{j=0}^{M-1}\left(z+a_{j}\right)
$$

and the function

$$
e(t)=\sum_{j=0}^{M-1} b_{j} e^{-a_{j} t}, \quad b_{j}=\frac{1}{P^{\prime}\left(-a_{j}\right)}=\frac{1}{\prod_{k=0, k \neq j}^{M-1}\left(-a_{j}+a_{k}\right)} .
$$

The coefficients $b_{j}$ appear in the partial fraction expansion

$$
\frac{1}{P(z)}=\sum_{j=0}^{M-1} b_{j} \frac{1}{z+a_{j}} \text {. }
$$

2010 Mathematics Subject Classification. Primary 46E39, Secondary 35K08.

Key words and phrases. discrete Sobolev inequality, discrete Laplacian, discrete heat kernel, Green's matrix, reproducing relation.

Received July 5, 2013; revised October 29, 2013. 
We assume $N=2,3,4, \ldots$ We set the indices of vertices as Figure 1 and introduce the discrete Laplacian $\boldsymbol{A}$ as

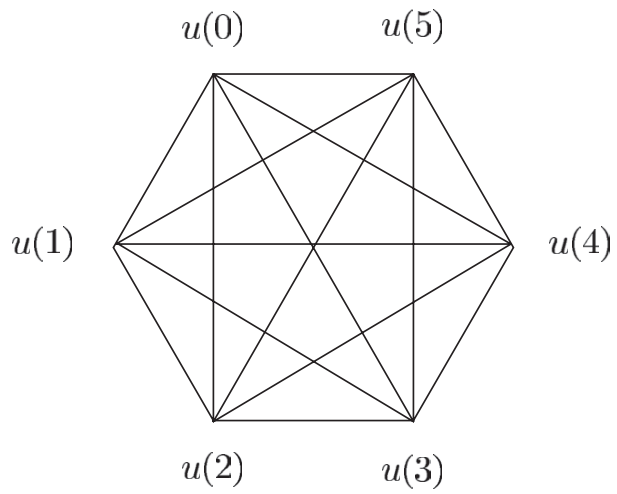

FiguRe 1. Complete graph $K_{6}$.

$$
\begin{aligned}
& \boldsymbol{A}=\boldsymbol{A}(N)=(a(N ; i, j)) \quad(0 \leq i, j \leq N-1), \\
& a(N ; i, j)= \begin{cases}N-1 & (i=j), \\
-1 & (i \neq j) .\end{cases}
\end{aligned}
$$

Here $\boldsymbol{A}$ is an $N \times N$ real symmetric positive-semidefinite matrix which has an eigenvalue 0 and whose eigenvector is $\mathbf{1}={ }^{t}(1,1, \ldots, 1)$. We introduce the constants $C_{0}, C_{0}(a)$ and $C_{1}(a)$ as

$$
\begin{aligned}
& C_{0}=\frac{N-1}{N^{M+1}}, \quad C_{0}(a)=\sum_{j=0}^{M-1} b_{j} \frac{a_{j}+1}{a_{j}\left(a_{j}+N\right)}, \\
& C_{1}(a)=\sum_{i, j=0}^{M-1} b_{i} b_{j} \frac{a_{i}+a_{j}+2}{\left(a_{i}+a_{j}\right)\left(a_{i}+a_{j}+2 N\right)} .
\end{aligned}
$$

For any $\quad \boldsymbol{u}={ }^{t}(u(0), u(1), \ldots, u(N-1)) \in \mathbf{C}^{N} \quad$ and $\quad \boldsymbol{u}(t)={ }^{t}(u(0, t), u(1, t), \ldots$, $u(N-1, t)) \in \mathbf{C}^{N}$ on the complete graph $K_{N}$, we define three kinds of the Sobolev energy:

$$
\begin{aligned}
& E(\boldsymbol{u})=\boldsymbol{u}^{*} \boldsymbol{A}^{M} \boldsymbol{u}, \quad E(a ; \boldsymbol{u})=\boldsymbol{u}^{*} \prod_{j=0}^{M-1}\left(\boldsymbol{A}+a_{j} \boldsymbol{I}\right) \boldsymbol{u}, \\
& F(a ; \boldsymbol{u}(t))=\int_{-\infty}^{\infty}\left\|\prod_{j=0}^{M-1}\left(\frac{d}{d t}+\boldsymbol{A}+a_{j} \boldsymbol{I}\right) \boldsymbol{u}(t)\right\|^{2} d t
\end{aligned}
$$

where $\|\boldsymbol{u}(t)\|^{2}=(\boldsymbol{u}(t))^{*} \boldsymbol{u}(t)$. We introduce the following three matrices 
(1.3) Discrete heat kernel: $\boldsymbol{H}(t)=\exp (-t \boldsymbol{A})$,

$$
\text { Green's matrix: } \boldsymbol{G}(a)=\left(\prod_{j=0}^{M-1}\left(\boldsymbol{A}+a_{j} \boldsymbol{I}\right)\right)^{-1}=\int_{0}^{\infty} e(t) \boldsymbol{H}(t) d t,
$$

(1.5) Pseudo Green's matrix: $\boldsymbol{G}_{*}=\lim _{a \rightarrow+0}\left(\boldsymbol{G}(a)-\prod_{j=0}^{M-1} a_{j}^{-1} \boldsymbol{E}_{0}\right)$,

where $\boldsymbol{E}_{0}=N^{-1} \mathbf{1}^{t} \mathbf{1}$ is a projection matrix to the eigenspace associated with the eigenvalue 0 of $\boldsymbol{A}$. In this paper, we obtain the best constants of three kinds of discrete Sobolev inequalities on $K_{N}$ as the following theorems.

THeOREM 1.1. For any $\boldsymbol{u} \in \mathbf{C}^{N}$ with ${ }^{t} \mathbf{1} \boldsymbol{u}=0$, there exists a positive constant $C$ which is independent of $\boldsymbol{u}$, such that the discrete Sobolev inequality

$$
\left(\max _{0 \leq j \leq N-1}|u(j)|\right)^{2} \leq C E(\boldsymbol{u})
$$

holds. Among such $C$, the best constant is $C_{0}$. If we replace $C$ by $C_{0}$ in (1.6), the equality holds iff $\boldsymbol{u}$ is parallel to any column of $\boldsymbol{G}_{*}$.

THEOREM 1.2. For any $\boldsymbol{u} \in \mathbf{C}^{N}$, there exists a positive constant $C$ which is independent of $\boldsymbol{u}$, such that the discrete Sobolev inequality

$$
\left(\max _{0 \leq j \leq N-1}|u(j)|\right)^{2} \leq C E(a ; \boldsymbol{u})
$$

holds. Among such $C$, the best constant is $C_{0}(a)$. If we replace $C$ by $C_{0}(a)$ in (1.7), the equality holds iff $\boldsymbol{u}$ is parallel to any column of $\boldsymbol{G}(a)$.

THEOREM 1.3. For any $\boldsymbol{u}(t) \in \mathbf{C}^{N}$, there exists a positive constant $C$ which is independent of $\boldsymbol{u}(t)$, such that the discrete Sobolev-type inequality

$$
\left(\sup _{0 \leq j \leq N-1,-\infty<s<\infty}|u(j, s)|\right)^{2} \leq C F(a ; \boldsymbol{u}(t))
$$

holds. Among such $C$, the best constant is $C_{1}(a)$. If we replace $C$ by $C_{1}(a)$ in (1.8), the equality holds iff $\boldsymbol{u}(t)$ is parallel to any column of

$$
\int_{|t|}^{\infty} \frac{1}{2} e\left(\frac{t+\sigma}{2}\right) e\left(\frac{t-\sigma}{2}\right) \boldsymbol{H}(\sigma) d \sigma \quad(-\infty<t<\infty) .
$$

Research on discrete Sobolev inequalities was performed in [1] on graphs, in our previous papers $[3,5]$ on periodic one-dimensional lattices and in $[2,4]$ on regular polyhedra. 
386 HIROYUKI YAMAGISHI, KOHTARO WATANABE AND YOSHINORI KAMETAKA

\section{Difference equations}

First, we explain three difference equations concerning the discrete heat kernel (1.3), the Green's matrix (1.4) and the pseudo Green's matrix (1.5).

Proposition 2.1. For any $\boldsymbol{f}(t) \in \mathbf{C}^{N}$, the discrete heat equation

$$
\prod_{j=0}^{M-1}\left(\frac{d}{d t}+\boldsymbol{A}+a_{j} \boldsymbol{I}\right) \boldsymbol{u}=\boldsymbol{f}(t) \quad(-\infty<t<\infty)
$$

has the unique solution given by

$$
\begin{aligned}
& \boldsymbol{u}(t)=\int_{-\infty}^{\infty} \boldsymbol{H}_{*}(t-s) \boldsymbol{f}(s) d s \quad(-\infty<t<\infty), \\
& \boldsymbol{H}_{*}(t)=Y(t) e(t) \boldsymbol{H}(t) \quad(-\infty<t<\infty),
\end{aligned}
$$

where $Y(t)=1(0 \leq t<\infty), 0(-\infty<t<0)$ is the Heaviside step function and $e(t)$ is defined in (1.1).

Proof of Proposition 2.1. By the Fourier transform

$$
\boldsymbol{u}(t) \hat{\rightarrow} \hat{\boldsymbol{u}}(\omega)=\int_{-\infty}^{\infty} e^{-\sqrt{-1} \omega t} \boldsymbol{u}(t) d t,
$$

(2.1) is transformed into

$$
\prod_{j=0}^{M-1}\left(\sqrt{-1} \omega+\boldsymbol{A}+a_{j} \boldsymbol{I}\right) \hat{\boldsymbol{u}}(\omega)=\hat{\boldsymbol{f}}(\omega) \quad(-\infty<\omega<\infty) .
$$

Solving this and remarking the formula (1.2), we have $\hat{\boldsymbol{u}}(\omega)=\hat{\boldsymbol{H}}_{*}(\omega) \hat{\boldsymbol{f}}(\omega)$, where

$$
\begin{aligned}
\hat{\boldsymbol{H}}_{*}(\omega) & =\left(\prod_{j=0}^{M-1}\left(\sqrt{-1} \omega \boldsymbol{I}+\boldsymbol{A}+a_{j} \boldsymbol{I}\right)\right)^{-1} \\
& =\sum_{j=0}^{M-1} b_{j}\left(\sqrt{-1} \omega \boldsymbol{I}+\boldsymbol{A}+a_{j} \boldsymbol{I}\right)^{-1}=\int_{-\infty}^{\infty} e^{-\sqrt{-1} \omega t} Y(t) e(t) \boldsymbol{H}(t) d t .
\end{aligned}
$$

From the inverse Fourier transform, we have (2.2) and (2.3). It should be noted that $\boldsymbol{H}_{*}(t)$ satisfies the relations:

$$
\begin{aligned}
& \left(\frac{d}{d t}+\boldsymbol{A}+a \boldsymbol{I}\right) \boldsymbol{H}_{*}=\boldsymbol{O}, \\
& \left.\boldsymbol{H}_{*}(t-s)\right|_{s=t-0}-\left.\boldsymbol{H}_{*}(t-s)\right|_{s=t+0}=\boldsymbol{I} \quad(-\infty<t<\infty),
\end{aligned}
$$

where $\boldsymbol{O}$ is zero matrix. This completes the proof of Proposition 2.1. 
Let us put $\omega$ as $\omega=\exp (\sqrt{-1} 2 \pi / N)$ and $\hat{a}(k)=0(k=0), N(1 \leq k \leq$ $N-1)$ be the eigenvalues of $\boldsymbol{A}$. Then, the Jordan canonical form of $\boldsymbol{A}$ is given by

$$
\hat{\boldsymbol{A}}=\operatorname{diag}\{\hat{a}(0), \hat{a}(1), \ldots, \hat{a}(N-1)\}=\operatorname{diag}\{0, N, \ldots, N\} .
$$

Further, $\boldsymbol{\varphi}_{k}(0 \leq k \leq N-1)$ denote the eigenvectors of $\boldsymbol{A}$ as follows:

$$
\boldsymbol{\varphi}_{k}=\frac{1}{\sqrt{N}}^{t}\left(1, \omega^{k}, \omega^{2 k}, \ldots, \omega^{(N-1) k}\right) \in \mathbf{C}^{N} \quad(0 \leq k \leq N-1) .
$$

These eigenvectors are chosen to satisfy the relation $\boldsymbol{\varphi}_{k}^{*} \boldsymbol{\varphi}_{l}=\delta(k-l)$, where $\delta(k)=1 \quad(k=0), \quad 0(k \neq 0)$. We introduce a unitary $N \times N$ matrix $\boldsymbol{W}=$ $\left(\boldsymbol{\varphi}_{0}, \ldots, \boldsymbol{\varphi}_{N-1}\right)$ and orthogonal projection matrices $\boldsymbol{E}_{k}=\boldsymbol{\varphi}_{k} \boldsymbol{\varphi}_{k}^{*}(0 \leq k \leq N-1)$. It is easy to see that the relations,

$$
\boldsymbol{W}^{*} \boldsymbol{W}=\boldsymbol{W} \boldsymbol{W}^{*}=\boldsymbol{I}, \quad \boldsymbol{E}_{k} \boldsymbol{E}_{l}=\delta(k-l) \boldsymbol{E}_{k}, \quad \boldsymbol{E}_{k}^{*}=\boldsymbol{E}_{k},
$$

hold. Using $\boldsymbol{E}_{k}$, we have the spectral decomposition of $\boldsymbol{I}$ and $\boldsymbol{A}$ as

$$
\begin{aligned}
\boldsymbol{I} & =\boldsymbol{W} \boldsymbol{W}^{*}=\sum_{k=0}^{N-1} \boldsymbol{\varphi}_{k} \boldsymbol{\varphi}_{k}^{*}=\sum_{k=0}^{N-1} \boldsymbol{E}_{k}, \\
\boldsymbol{A} & =\boldsymbol{W} \hat{\boldsymbol{A}} \boldsymbol{W}^{*}=\sum_{k=0}^{N-1} \hat{a}(k) \boldsymbol{\varphi}_{k} \boldsymbol{\varphi}_{k}^{*}=\sum_{k=0}^{N-1} \hat{a}(k) \boldsymbol{E}_{k}=N \sum_{k=1}^{N-1} \boldsymbol{E}_{k} \\
& =N\left(\boldsymbol{I}-\boldsymbol{E}_{0}\right) .
\end{aligned}
$$

Using $\boldsymbol{E}_{k} \boldsymbol{E}_{l}=\delta(k-l) \boldsymbol{E}_{k}$, we have

$$
\begin{aligned}
& \boldsymbol{A}^{M}=N^{M} \sum_{k=1}^{N-1} \boldsymbol{E}_{k}=N^{M}\left(\boldsymbol{I}-\boldsymbol{E}_{0}\right), \\
& \prod_{j=0}^{M-1}\left(\boldsymbol{A}+a_{j} \boldsymbol{I}\right)=\prod_{j=0}^{M-1} \sum_{k=0}^{N-1}\left(\hat{a}(k)+a_{j}\right) \boldsymbol{E}_{k}=\sum_{k=0}^{N-1} P(\hat{a}(k)) \boldsymbol{E}_{k} .
\end{aligned}
$$

Proposition 2.2. For any $\boldsymbol{f} \in \mathbf{C}^{N}$, the difference equation

$$
\prod_{j=0}^{M-1}\left(\boldsymbol{A}+a_{j} \boldsymbol{I}\right) \boldsymbol{u}=\boldsymbol{f}
$$

has the unique solution given by $\boldsymbol{u}=\boldsymbol{G} \boldsymbol{f}$, where $\boldsymbol{G}=\boldsymbol{G}(a)$ is the Green's matrix expressed as

$$
\boldsymbol{G}=\sum_{j=0}^{M-1} b_{j}\left[\frac{1}{a_{j}+N} \boldsymbol{I}+\left(\frac{1}{a_{j}}-\frac{1}{a_{j}+N}\right) \boldsymbol{E}_{0}\right]
$$


Proof of Proposition 2.2. From (2.4) and (2.6), we have

$$
\sum_{k=0}^{N-1} \boldsymbol{E}_{k} \boldsymbol{f}=\boldsymbol{I} \boldsymbol{f}=\boldsymbol{f}=\prod_{j=0}^{M-1}\left(\boldsymbol{A}+a_{j} \boldsymbol{I}\right) \boldsymbol{u}=\sum_{k=0}^{N-1} P(\hat{a}(k)) \boldsymbol{E}_{k} \boldsymbol{u} .
$$

Multiplying $\boldsymbol{E}_{l}$ from the left to both sides of the above relation and using the relation $\boldsymbol{E}_{k} \boldsymbol{E}_{l}=\delta(k-l) \boldsymbol{E}_{k}$, we obtain $\boldsymbol{E}_{l} \boldsymbol{u}=(P(\hat{a}(l)))^{-1} \boldsymbol{E}_{l} \boldsymbol{f}$. Then, we see that

$$
\begin{aligned}
\boldsymbol{u} & =\boldsymbol{I} \boldsymbol{u}=\sum_{l=0}^{N-1} \boldsymbol{E}_{l} \boldsymbol{u}=\sum_{l=0}^{N-1} \frac{1}{P(\hat{a}(l))} \boldsymbol{E}_{l} \boldsymbol{f}=\boldsymbol{G} \boldsymbol{f} \\
\boldsymbol{G} & =\sum_{l=0}^{N-1} \frac{1}{P(\hat{a}(l))} \boldsymbol{E}_{l}=\frac{1}{P(0)} \boldsymbol{E}_{0}+\frac{1}{P(N)} \sum_{l=1}^{N-1} \boldsymbol{E}_{l} \\
& =\frac{1}{P(N)} \boldsymbol{I}+\left(\frac{1}{P(0)}-\frac{1}{P(N)}\right) \boldsymbol{E}_{0} .
\end{aligned}
$$

Using (1.2), we have (2.7). This completes the proof of Proposition 2.2.

Proposition 2.3. For any $\boldsymbol{f} \in \mathbf{C}^{N}$ with the solvability condition ${ }^{t} \mathbf{1} \boldsymbol{f}=0$, the difference equation $\boldsymbol{A}^{M} \boldsymbol{u}=\boldsymbol{f}$ with the orthogonality condition ${ }^{t} \mathbf{1} \boldsymbol{u}=0$ has the unique solution given by $\boldsymbol{u}=\boldsymbol{G}_{*} \boldsymbol{f}$, where $\boldsymbol{G}_{*}$ is the pseudo Green's matrix expressed as

$$
\boldsymbol{G}_{*}=\frac{1}{N^{M}}\left(\boldsymbol{I}-\boldsymbol{E}_{0}\right)=\frac{1}{N^{M}} \sum_{k=1}^{N-1} \boldsymbol{E}_{k} .
$$

$\boldsymbol{G}_{*}$ satisfies $\boldsymbol{A}^{M} \boldsymbol{G}_{*}=\boldsymbol{G}_{*} \boldsymbol{A}^{M}=\boldsymbol{I}-\boldsymbol{E}_{0}, \boldsymbol{G}_{*} \boldsymbol{E}_{0}=\boldsymbol{E}_{0} \boldsymbol{G}_{*}=\boldsymbol{O}$.

Proof of Proposition 2.3. From (2.4), (2.5) and $\boldsymbol{E}_{0} \boldsymbol{f}=N^{-1} \mathbf{1}^{t} \mathbf{1} \boldsymbol{f}=\mathbf{0}$, where 0 is zero vector, we have

$$
\sum_{k=1}^{N-1} \boldsymbol{E}_{k} \boldsymbol{f}=\sum_{k=0}^{N-1} \boldsymbol{E}_{k} \boldsymbol{f}=\boldsymbol{I} \boldsymbol{f}=\boldsymbol{f}=\boldsymbol{A}^{M} \boldsymbol{u}=N^{M} \sum_{k=1}^{N-1} \boldsymbol{E}_{k} \boldsymbol{u} .
$$

Multiplying $\boldsymbol{E}_{l}$ from the left to both sides of the above relation and using the relation $\boldsymbol{E}_{k} \boldsymbol{E}_{l}=\delta(k-l) \boldsymbol{E}_{k}$, we obtain $\boldsymbol{E}_{l} \boldsymbol{u}=N^{-M} \boldsymbol{E}_{l} \boldsymbol{f}(1 \leq l \leq N-1)$. Then, using $\boldsymbol{E}_{0} \boldsymbol{u}=N^{-1} \mathbf{1}^{t} \mathbf{1} \boldsymbol{u}=\mathbf{0}$, we see that

$$
\boldsymbol{u}=\boldsymbol{I} \boldsymbol{u}=\sum_{l=0}^{N-1} \boldsymbol{E}_{l} \boldsymbol{u}=\sum_{l=1}^{N-1} \boldsymbol{E}_{l} \boldsymbol{u}=\frac{1}{N^{M}} \sum_{l=1}^{N-1} \boldsymbol{E}_{l} \boldsymbol{f}=\boldsymbol{G}_{*} \boldsymbol{f} .
$$

So we have (2.8). Moreover, using (2.8) and $\boldsymbol{E}_{k} \boldsymbol{E}_{l}=\delta(k-l) \boldsymbol{E}_{k}$, we have 


$$
\begin{aligned}
& \boldsymbol{A}^{M} \boldsymbol{G}_{*}=\sum_{k, l=1}^{N-1} N^{M} \boldsymbol{E}_{k} \frac{1}{N^{M}} \boldsymbol{E}_{l}=\sum_{k=1}^{N-1} \boldsymbol{E}_{k}=\boldsymbol{I}-\boldsymbol{E}_{0}, \\
& \boldsymbol{G}_{*} \boldsymbol{E}_{0}=\sum_{k=1}^{N-1} \frac{1}{N^{M}} \boldsymbol{E}_{k} \boldsymbol{E}_{0}=\boldsymbol{O} .
\end{aligned}
$$

From the above relations, we see that $\boldsymbol{G}_{*}$ is a Penrose-Moore generalized inverse matrix of $\boldsymbol{A}^{M}$. This completes the proof of Proposition 2.3.

Next, we compute $\boldsymbol{H}, \boldsymbol{G}$ and $\boldsymbol{G}_{*}$ which represent the best constants of Sobolev inequalities. We introduce the $N$-dimension vector

$$
\boldsymbol{\delta}_{j}={ }^{t}(\delta(i-j) \quad)_{0 \leq i \leq N-1} .
$$

LEMMA 2.1. For any fixed $j(0 \leq j \leq N-1)$, we have the following relations:

$$
\begin{aligned}
& { }^{t} \boldsymbol{\delta}_{j} \boldsymbol{H}(t) \boldsymbol{\delta}_{j}=\frac{1}{N}\left(1+(N-1) e^{-N t}\right) . \\
& \int_{-\infty}^{\infty}\left\|\boldsymbol{H}_{*}(t) \boldsymbol{\delta}_{j}\right\|^{2} d t=C_{1}(a) . \\
& { }^{t} \boldsymbol{\delta}_{j} \boldsymbol{G} \boldsymbol{\delta}_{j}=C_{0}(a) . \\
& { }^{t} \boldsymbol{\delta}_{j} \boldsymbol{G}_{*} \boldsymbol{\delta}_{j}=C_{0} .
\end{aligned}
$$

Proof of Lemma 2.1. Applying $\boldsymbol{A}^{j}=N^{j}\left(\boldsymbol{I}-\boldsymbol{E}_{0}\right)(j=1,2,3, \ldots)$ in $(2.5)$ to (1.3), we have

$$
\begin{aligned}
\boldsymbol{H}(t) & =\exp (-t \boldsymbol{A})=\sum_{j=0}^{\infty} \frac{1}{j !}(-t)^{j} \boldsymbol{A}^{j}=\boldsymbol{I}+\sum_{j=1}^{\infty} \frac{1}{j !}(-t)^{j} \boldsymbol{A}^{j} \\
& =\boldsymbol{I}+\left(\sum_{j=1}^{\infty} \frac{1}{j !}(-t N)^{j}\right)\left(\boldsymbol{I}-\boldsymbol{E}_{0}\right)=\boldsymbol{I}+\left(-1+e^{-N t}\right)\left(\boldsymbol{I}-\boldsymbol{E}_{0}\right) .
\end{aligned}
$$

From the relation above, we have

$$
\boldsymbol{H}(t)=e^{-N t} \boldsymbol{I}+\left(1-e^{-N t}\right) \boldsymbol{E}_{0}
$$

and (2.9). Noting ${ }^{t} \boldsymbol{H}(t)=\boldsymbol{H}(t),(\boldsymbol{H}(t))^{2}=\boldsymbol{H}(2 t)$ and (2.3), we have

$$
\begin{aligned}
\int_{-\infty}^{\infty}\left\|\boldsymbol{H}_{*}(t) \boldsymbol{\delta}_{j}\right\|^{2} d t & =\int_{-\infty}^{\infty}{ }^{t}\left(\boldsymbol{H}_{*}(t) \boldsymbol{\delta}_{j}\right)\left(\boldsymbol{H}_{*}(t) \boldsymbol{\delta}_{j}\right) d t \\
& =\int_{-\infty}^{\infty}{ }^{t} \boldsymbol{\delta}_{j}\left(\boldsymbol{H}_{*}(t)\right)^{2} \boldsymbol{\delta}_{j} d t=\int_{0}^{\infty} e^{2}(t)^{t} \boldsymbol{\delta}_{j} \boldsymbol{H}(2 t) \boldsymbol{\delta}_{j} d t
\end{aligned}
$$




$$
\begin{aligned}
& =\int_{0}^{\infty} \sum_{i, j=0}^{M-1} b_{i} b_{j} e^{-\left(a_{i}+a_{j}\right) t} \frac{1}{N}\left(1+(N-1) e^{-2 N t}\right) d t \\
& =\frac{1}{N} \sum_{i, j=0}^{M-1} b_{i} b_{j} \int_{0}^{\infty}\left[e^{-\left(a_{i}+a_{j}\right) t}+(N-1) e^{-\left(a_{i}+a_{j}+2 N\right) t}\right] d t \\
& =\frac{1}{N} \sum_{i, j=0}^{M-1} b_{i} b_{j}\left[\frac{1}{a_{i}+a_{j}}+(N-1) \frac{1}{a_{i}+a_{j}+2 N}\right]=C_{1}(a) .
\end{aligned}
$$

So we have (2.10). Since the proofs of (2.11) and (2.12) are standard and easy, we omit it. This completes the proof of Lemma 2.1 .

\section{Reproducing relation}

For $\boldsymbol{u}, \boldsymbol{v} \in \mathbf{C}^{N}$, we introduce the inner products

$$
\begin{aligned}
& (\boldsymbol{u}, \boldsymbol{v})=\boldsymbol{v}^{*} \boldsymbol{u}, \\
& \|\boldsymbol{u}\|^{2}=(\boldsymbol{u}, \boldsymbol{u}), \\
& (\boldsymbol{u}, \boldsymbol{v})_{H}=\left(\prod_{j=0}^{M-1}\left(\boldsymbol{A}+a_{j} \boldsymbol{I}\right) \boldsymbol{u}, \boldsymbol{v}\right)=\boldsymbol{v}^{*} \prod_{j=0}^{M-1}\left(\boldsymbol{A}+a_{j} \boldsymbol{I}\right) \boldsymbol{u}, \\
& \|\boldsymbol{u}\|_{H}^{2}=(\boldsymbol{u}, \boldsymbol{u})_{H}=E(a ; \boldsymbol{u}) .
\end{aligned}
$$

For $\boldsymbol{u}, \boldsymbol{v} \in \mathbf{C}_{0}^{N}:=\left\{\boldsymbol{x} \mid \boldsymbol{x} \in \mathbf{C}^{N}\right.$ and $\left.{ }^{t} \mathbf{1} \boldsymbol{x}=0\right\}$, we introduce the inner product

$$
\begin{aligned}
& (\boldsymbol{u}, \boldsymbol{v})_{A}=\left(\boldsymbol{A}^{M} \boldsymbol{u}, \boldsymbol{v}\right)=\boldsymbol{v}^{*} \boldsymbol{A}^{M} \boldsymbol{u}, \\
& \|\boldsymbol{u}\|_{A}^{2}=(\boldsymbol{u}, \boldsymbol{u})_{A}=E(\boldsymbol{u}) .
\end{aligned}
$$

First, we show the positive definiteness of $(\cdot, \cdot)_{H}$ and $(\cdot, \cdot)_{A}$.

Lemma 3.1.

(1) For $\boldsymbol{u}, \boldsymbol{v} \in \mathbf{C}^{N},(\boldsymbol{u}, \boldsymbol{v})_{H}$ is defined as an inner product.

(2) For $\boldsymbol{u}, \boldsymbol{v} \in \mathbf{C}_{0}^{N},(\boldsymbol{u}, \boldsymbol{v})_{A}$ is defined as an inner product.

Proof of Lemma 3.1. (1) is obvious since $\left(\boldsymbol{A}+a_{0} \boldsymbol{I}\right) \cdots\left(\boldsymbol{A}+a_{M-1} \boldsymbol{I}\right)$ is positive definite. We show only (2). For $\boldsymbol{u} \in \mathbf{C}_{0}^{N}$, we have

$$
\begin{aligned}
& \boldsymbol{u}=\boldsymbol{I} \boldsymbol{u}=\sum_{k=0}^{N-1} \boldsymbol{E}_{k} \boldsymbol{u}=\sum_{k=1}^{N-1} \boldsymbol{E}_{k} \boldsymbol{u}, \\
& \|\boldsymbol{u}\|^{2}=\sum_{k, l=0}^{N-1} \boldsymbol{u}^{*} \boldsymbol{E}_{l}^{*} \boldsymbol{E}_{k} \boldsymbol{u}=\sum_{k=1}^{N-1}\left\|\boldsymbol{E}_{k} \boldsymbol{u}\right\|^{2} .
\end{aligned}
$$


From the relation $\boldsymbol{E}_{k}=\boldsymbol{E}_{k} \boldsymbol{E}_{k}=\boldsymbol{E}_{k}^{*} \boldsymbol{E}_{k}$, we have

$$
\|\boldsymbol{u}\|_{A}^{2}=\boldsymbol{u}^{*} \boldsymbol{A}^{M} \boldsymbol{u}=N^{M} \sum_{k=1}^{N-1} \boldsymbol{u}^{*} \boldsymbol{E}_{k}^{*} \boldsymbol{E}_{k} \boldsymbol{u}=N^{M} \sum_{k=1}^{N-1}\left\|\boldsymbol{E}_{k} \boldsymbol{u}\right\|^{2}=N^{M}\|\boldsymbol{u}\|^{2} .
$$

Since $N>0$, we have $\|\boldsymbol{u}\|_{A}^{2} \geq 0$, and $\|\boldsymbol{u}\|_{A}^{2}=0$ holds iff $\boldsymbol{u}=\mathbf{0}$. This completes the proof of Lemma 3.1 .

Next, we show that $\boldsymbol{G}$ and $\boldsymbol{G}_{*}$ are a reproducing matrix for the inner products $(\cdot, \cdot)_{H}$ and $(\cdot, \cdot)_{A}$, respectively.

Lemma 3.2. For any $\boldsymbol{u} \in \mathbf{C}_{0}^{N}$ and fixed $j(0 \leq j \leq N-1)$, we have the following reproducing relations:

(1) $u(j)=\left(\boldsymbol{u}, \boldsymbol{G}_{*} \boldsymbol{\delta}_{j}\right)_{A}$.

(2) $C_{0}={ }^{t} \boldsymbol{\delta}_{j} \boldsymbol{G}_{*} \boldsymbol{\delta}_{j}=\left\|\boldsymbol{G}_{*} \boldsymbol{\delta}_{j}\right\|_{A}^{2}=E\left(\boldsymbol{G}_{*} \boldsymbol{\delta}_{j}\right)$.

Proof of Lemma 3.2. Noting $\boldsymbol{G}_{*}^{*}=\boldsymbol{G}_{*}$, we have (1) as follows:

$$
\left(\boldsymbol{u}, \boldsymbol{G}_{*} \boldsymbol{\delta}_{j}\right)_{A}={ }^{t} \boldsymbol{\delta}_{j} \boldsymbol{G}_{*} \boldsymbol{A}^{M} \boldsymbol{u}={ }^{t} \boldsymbol{\delta}_{j}\left(\boldsymbol{I}-\boldsymbol{E}_{0}\right) \boldsymbol{u}={ }^{t} \boldsymbol{\delta}_{j} \boldsymbol{u}-\frac{1}{N} \mathbf{1}^{t} \mathbf{1} \boldsymbol{u}=u(j) .
$$

Putting $\boldsymbol{u}=\boldsymbol{G}_{*} \boldsymbol{\delta}_{j}$ in (1) and using (2.12), we obtain (2).

Lemma 3.3. For any $\boldsymbol{u} \in \mathbf{C}^{N}$ and fixed $j(0 \leq j \leq N-1)$, we have the following reproducing relations:

(1) $u(j)=\left(\boldsymbol{u}, \boldsymbol{G} \boldsymbol{\delta}_{j}\right)_{H}$.

(2) $C_{0}(a)={ }^{t} \boldsymbol{\delta}_{j} \boldsymbol{G} \boldsymbol{\delta}_{j}=\left\|\boldsymbol{G} \boldsymbol{\delta}_{j}\right\|_{H}^{2}=E\left(a ; \boldsymbol{G} \boldsymbol{\delta}_{j}\right)$.

The proof of Lemma 3.3. Noting $\boldsymbol{G}^{*}=\boldsymbol{G}$, we have (1) as follows:

$$
\left(\boldsymbol{u}, \boldsymbol{G} \boldsymbol{\delta}_{j}\right)_{H}={ }^{t} \boldsymbol{\delta}_{j} \boldsymbol{G} \prod_{j=0}^{M-1}\left(\boldsymbol{A}+a_{j} \boldsymbol{I}\right) \boldsymbol{u}={ }^{t} \boldsymbol{\delta}_{j} \boldsymbol{I} \boldsymbol{u}=u(j) .
$$

Putting $\boldsymbol{u}=\boldsymbol{G} \boldsymbol{\delta}_{j}$ in (1) and using (2.11), we obtain (2).

\section{Proof of theorems}

This section is devoted to the proof of main theorems.

Proof of Theorem 1.1. Applying the Schwarz inequality to Lemma 3.2 (1) and using Lemma 3.2 (2), we have

$$
|u(j)|^{2} \leq\|\boldsymbol{u}\|_{A}^{2}\left\|\boldsymbol{G}_{*} \boldsymbol{\delta}_{j}\right\|_{A}^{2}=C_{0} E(\boldsymbol{u}) .
$$


Taking the maximum with respect to $j$ on both sides, we obtain the discrete Sobolev inequality

$$
\left(\max _{0 \leq j \leq N-1}|u(j)|\right)^{2} \leq C_{0} E(\boldsymbol{u}) .
$$

For any fixed number $j_{0}\left(0 \leq j_{0} \leq N-1\right)$, if we take $\boldsymbol{u}=\boldsymbol{G}_{*} \boldsymbol{\delta}_{j_{0}}$ in (4.1), then we have

$$
\left(\max _{0 \leq j \leq N-1}\left|{ }^{t} \boldsymbol{\delta}_{j} \boldsymbol{G}_{*} \boldsymbol{\delta}_{j_{0}}\right|\right)^{2} \leq C_{0} E\left(\boldsymbol{G}_{*} \boldsymbol{\delta}_{j_{0}}\right)=\left(C_{0}\right)^{2} .
$$

Combining this with the trivial inequality

$$
\left(C_{0}\right)^{2}=\left|{ }^{t} \boldsymbol{\delta}_{j_{0}} \boldsymbol{G}_{*} \boldsymbol{\delta}_{j_{0}}\right|^{2} \leq\left(\max _{0 \leq j \leq N-1}\left|{ }^{t} \boldsymbol{\delta}_{j} \boldsymbol{G}_{*} \boldsymbol{\delta}_{j_{0}}\right|\right)^{2},
$$

we have

$$
\left(\max _{0 \leq j \leq N-1}\left|{ }^{t} \boldsymbol{\delta}_{j} \boldsymbol{G}_{*} \boldsymbol{\delta}_{j_{0}}\right|\right)^{2}=C_{0} E\left(\boldsymbol{G}_{*} \boldsymbol{\delta}_{j_{0}}\right) .
$$

This shows that $C_{0}$ is the best constant of (4.1) and the equality holds for any column of $\boldsymbol{G}_{*}$. This completes the proof of Theorem 1.1 .

Proof of Theorem 1.2. Applying the Schwarz inequality to Lemma 3.3 (1) and using Lemma 3.3 (2), we have

$$
|u(j)|^{2} \leq\|\boldsymbol{u}\|_{H}^{2}\left\|\boldsymbol{G} \boldsymbol{\delta}_{j}\right\|_{H}^{2}=C_{0}(a) E(a ; \boldsymbol{u}) .
$$

Taking the maximum with respect to $j$ on both sides, we have the discrete Sobolev inequality

$$
\left(\max _{0 \leq j \leq N-1}|u(j)|\right)^{2} \leq C_{0}(a) E(a ; \boldsymbol{u}) .
$$

For any fixed number $j_{0}\left(0 \leq j_{0} \leq N-1\right)$, if we take $\boldsymbol{u}=\boldsymbol{G} \boldsymbol{\delta}_{j_{0}}$ in (4.2), then we have

$$
\left(\max _{0 \leq j \leq N-1}\left|{ }^{t} \boldsymbol{\delta}_{j} \boldsymbol{G} \boldsymbol{\delta}_{j_{0}}\right|\right)^{2} \leq C_{0}(a) E\left(a ; \boldsymbol{G} \boldsymbol{\delta}_{j_{0}}\right)=\left(C_{0}(a)\right)^{2} .
$$

Combining this with the trivial inequality

$$
\left(C_{0}(a)\right)^{2}=\left|{ }^{t} \boldsymbol{\delta}_{j_{0}} \boldsymbol{G} \boldsymbol{\delta}_{j_{0}}\right|^{2} \leq\left(\max _{0 \leq j \leq N-1}\left|{ }^{t} \boldsymbol{\delta}_{j} \boldsymbol{G} \boldsymbol{\delta}_{j_{0}}\right|\right)^{2},
$$

we have

$$
\left(\max _{0 \leq j \leq N-1}\left|{ }^{t} \boldsymbol{\delta}_{j} \boldsymbol{G} \boldsymbol{\delta}_{j_{0}}\right|\right)^{2}=C_{0}(a) E\left(a ; \boldsymbol{G} \boldsymbol{\delta}_{j_{0}}\right)
$$


This shows that $C_{0}(a)$ is the best constant of (4.2) and the equality holds for any column of $\boldsymbol{G}$. This completes the proof of Theorem 1.2.

Proof of Theorem 1.3. Replacing $t$ with $s$ in (2.2), we have

$$
\boldsymbol{u}(s)=\int_{-\infty}^{\infty} \boldsymbol{H}_{*}(s-t) \boldsymbol{f}(t) d t,
$$

or equivalently

$$
\begin{aligned}
u(j, s) & ={ }^{t} \boldsymbol{\delta}_{j} \boldsymbol{u}(s) \\
& =\int_{-\infty}^{\infty}{ }^{t} \boldsymbol{\delta}_{j} \boldsymbol{H}_{*}(s-t) \boldsymbol{f}(t) d t=\int_{-\infty}^{\infty}{ }^{t}\left(\boldsymbol{H}_{*}(s-t) \boldsymbol{\delta}_{j}\right) \boldsymbol{f}(t) d t .
\end{aligned}
$$

Applying the Schwarz inequality to (4.3), we have

$$
\begin{aligned}
|u(j, s)|^{2} & \leq \int_{-\infty}^{\infty}\left\|\boldsymbol{H}_{*}(s-t) \boldsymbol{\delta}_{j}\right\|^{2} d t \int_{-\infty}^{\infty}\|\boldsymbol{f}(t)\|^{2} d t \\
& =\int_{-\infty}^{\infty}\left\|\boldsymbol{H}_{*}(t) \boldsymbol{\delta}_{j}\right\|^{2} d t \int_{-\infty}^{\infty}\left\|\prod_{j=0}^{M-1}\left(\frac{d}{d t}+\boldsymbol{A}+a_{j} \boldsymbol{I}\right) \boldsymbol{u}(t)\right\|^{2} d t \\
& =C_{1}(a) F(a ; \boldsymbol{u}(t)),
\end{aligned}
$$

where we use (2.1) and (2.10). Taking the supremum with respect to $j$ and $s$, we obtain the discrete Sobolev-type inequality

$$
\left(\sup _{0 \leq j \leq N-1,-\infty<s<\infty}|u(j, s)|\right)^{2} \leq C_{1}(a) F(a ; \boldsymbol{u}(t)) .
$$

For any fixed number $j_{0}\left(0 \leq j_{0} \leq N-1\right)$, we introduce the vector $\boldsymbol{U}(t)$ defined by

$$
\begin{aligned}
& \boldsymbol{U}(t)=\int_{-\infty}^{\infty} \boldsymbol{H}_{*}(t-s) \boldsymbol{H}_{*}(-s) \boldsymbol{\delta}_{j_{0}} d s \\
& U(j, t)={ }^{t} \boldsymbol{\delta}_{j} \boldsymbol{U}(t)=\int_{-\infty}^{\infty}{ }^{t} \boldsymbol{\delta}_{j} \boldsymbol{H}_{*}(t-s) \boldsymbol{H}_{*}(-s) \boldsymbol{\delta}_{j_{0}} d s .
\end{aligned}
$$

Then we have

$$
\begin{aligned}
\left(\sup _{0 \leq j \leq N-1,-\infty<s<\infty}|U(j, s)|\right)^{2} & \leq C_{1}(a) F(a ; \boldsymbol{U}(t)) \\
& =C_{1}(a) \int_{-\infty}^{\infty}\left\|\prod_{j=0}^{M-1}\left(\frac{d}{d t}+\boldsymbol{A}+a_{j} \boldsymbol{I}\right) \boldsymbol{U}(t)\right\|^{2} d t \\
& =C_{1}(a) \int_{-\infty}^{\infty}\left\|\boldsymbol{H}_{*}(-t) \boldsymbol{\delta}_{j_{0}}\right\|^{2} d t=\left(C_{1}(a)\right)^{2}
\end{aligned}
$$




\section{HIROYUKI YAMAGISHI, KOHTARO WATANABE AND YOSHINORI KAMETAKA}

Combining this with the trivial inequality

$$
\left(C_{1}(a)\right)^{2}=\left|U\left(j_{0}, 0\right)\right|^{2} \leq\left(\sup _{0 \leq j \leq N-1,-\infty<s<\infty}|U(j, s)|\right)^{2},
$$

we have

$$
\left(\sup _{0 \leq j \leq N-1,-\infty<s<\infty}|U(j, s)|\right)^{2}=C_{1}(a) F(a ; \boldsymbol{U}(t)) .
$$

This shows that $C_{1}(a)$ is the best constant of (4.4) and the equality holds for $\boldsymbol{u}(t)=\boldsymbol{U}(t)$. From (4.5), we have (1.9) as follows:

$$
\begin{aligned}
\boldsymbol{U}(t) & =\int_{-\infty}^{\infty} \boldsymbol{H}_{*}(t-s) \boldsymbol{H}_{*}(-s) \boldsymbol{\delta}_{j_{0}} d s \\
& =\int_{-\infty}^{\infty} Y(t-s) e(t-s) \boldsymbol{H}(t-s) Y(-s) e(-s) \boldsymbol{H}(-s) \boldsymbol{\delta}_{j_{0}} d s \\
& =\int_{-\infty}^{0 \wedge t} e(t-s) e(-s) \boldsymbol{H}(t-2 s) \boldsymbol{\delta}_{j_{0}} d s \\
& =\int_{|t|}^{\infty} \frac{1}{2} e\left(\frac{t+\sigma}{2}\right) e\left(\frac{t-\sigma}{2}\right) \boldsymbol{H}(\sigma) \boldsymbol{\delta}_{j_{0}} d \sigma .
\end{aligned}
$$

This completes the proof of Theorem 1.3.

\section{REFERENCES}

[1] F. R. K. Chung AND S.-T. Yau, Eigenvalues of graphs and Sobolev inequalities, Combin. Probab. Comput. 4 (1995), 11-25.

[2] Y. Kametaka, K. Watanabe, H. Yamagishi, A. Nagai and K. Takemura, The best constant of discrete Sobolev inequality on regular polyhedron, Transactions of the Japan Society for Industrial and Applied Mathematics 21 (2011), 289-308 (in Japanese).

[ 3 ] A. Nagai, Y. Kametaka, H. Yamagishi, K. Takemura and K. Watanabe, Discrete Bernoulli polynomials and the best constant of discrete Sobolev inequality, Funkcial. Ekvac. 51 (2008), 307-327.

[ 4 ] H. Yamagishi, Y. Kametaka, A. Nagai, K. Watanabe and K. Takemura, The best constant of three kinds of discrete Sobolev inequalities on regular polyhedron, Tokyo J. Math. 36 (2013), 253-268.

[ 5 ] H. Yamagishi, A. Nagai, K. Watanabe, K. Takemura and Y. Kametaka, The best constant of discrete Sobolev inequality corresponding to a bending problem of a string, Kumamoto J. Math. 25 (2012), 1-15.

\section{Hiroyuki Yamagishi}

Tokyo Metropolitan College of Industrial Technology

1-10-40 Higashi-OI, Shinagawa

TOKYO 140-0011

JAPAN

E-mail: yamagisi@s.metro-cit.ac.jp 
Kohtaro Watanabe

Department of Computer Science

National Defense ACADEMY

1-10-20 YoKOSUKA

KANAGAWA 239-8686

JAPAN

E-mail: wata@nda.ac.jp

Yoshinori Kametaka

Faculty of Engineering Science, Osaka University

1-3 MachiKaneyama-ChO, TOYONAKA

OSAKA 560-8531

JAPAN

E-mail: kametaka@sigmath.es.osaka-u.ac.jp 